\title{
Why have no transiting planets been found in star clusters?
}

\author{
Kenneth Janes and Ji-Hyun Kim \\ Boston University, \\ Astronomy Department, 725 Commonwealth Ave, Boston, MA 02215 \\ email: janes@bu.edu, jhkim26@bu.edu
}

\begin{abstract}
Since the suggestion by Janes 1996 that star clusters might be attractive targets to search for transiting planets, there have been more than 20 searches in both open and globular clusters. To date, no confirmed hot Jupiter transiting planets have been found in clusters. We review the statistics for finding short-period planets and we summarize the open cluster work to date, including our own (so far negative) search in NGC 7789. While individually, the negative results are not surprising, the consistent failure to find planets in clusters may suggest differences in the formation or evolution of planetary systems in clusters.
\end{abstract}

\section{Planets in clusters}

Star clusters would seem to make ideal targets for transiting planets searches (Janes 1996). Yet no transiting planets have actually been confirmed as members of star clusters, in spite of a number of studies. There are (at least) three possible explanations: 1) globular clusters especially are typically metal-poor, relative to the Doppler-discovered planetary systems; 2) the internal dynamics of clusters may prevent the formation of planets, or hasten their destruction; 3) there may simply be too few open cluster members to make the probability for finding a transiting planet likely.

Table 1 shows the result of a literature search for open cluster transit studies. We obtained secondary information on the clusters listed in the table from the WEBDA database (http://www.univie.ac.at/webda/). We did not include globular clusters in our survey, but we note that in spite of several intensive searches in globulars (e.g., Gilliland et al. (2000)), no planets have been found. While some of the candidates noted in the table may be transiting planets, they are probably not cluster members, simply because most of the stars surveyed in the studies listed in Table 1 are non-members.

So how many planets might we expect to find? Fischer \& Valenti (2005) showed that, as a function of metallicity, the probability for a star to have a large planet with a period up to 40 days is $P_{\text {planet }}=0.03 \times 10^{2.0[\mathrm{Fe} / \mathrm{H}]}$. About $17.5 \%$ of the Fischer-Valenti sample are hot Jupiters with periods $\leqslant 4$ days, so the differential probability per day for hot Jupiters is roughly

$$
P_{H J}=0.0013 \times 10^{2.0[\mathrm{Fe} / \mathrm{H}]} d p .
$$

The geometrical probability that a planet will transit (ignoring its size) is $P_{T}=R_{*} / a_{p}$, where $R_{*}$ is the radius of the star (in A.U.) and $a_{p}$ is the radius of the planet's orbit. Using Kepler's law (and now ignoring the mass of the planet), this becomes

$$
P_{T}=R_{*} M_{*}^{-1 / 3} p^{-2 / 3},
$$

where $M_{*}$ is in solar masses, $p$ is in years. 
Table 1. Published open cluster transit searches.

\begin{tabular}{|c|c|c|c|c|c|c|}
\hline Cluster & $\begin{array}{l}\text { \# of } \\
\text { stars }\end{array}$ & $\begin{array}{c}\text { Age } \\
{[\text { Gyr }]}\end{array}$ & $\begin{array}{l}\text { Nights } \\
\text { observed }\end{array}$ & $\begin{array}{c}\text { Hours } \\
\text { observed }\end{array}$ & $\begin{array}{c}\text { Results } \\
\text { (\# of candidates) }\end{array}$ & References \\
\hline NGC 1245 & 870 & 1.04 & 19 & 78 & 6 & Burke et al. (2006) \\
\hline NGC 2099 & - & 0.32 & - & - & In progress & Burke (2008) (STEPSS); WEBDA \\
\hline NGC 2158 & 5159 & 2.00 & 59 & 260 & 1 & Mochejska et al. (2006) \\
\hline NGC 2301 & 4000 & 0.16 & 12 & 70 & None & Howell et al. (2005) \\
\hline NGC 2362 & 475 & 0.005 & 18 & 100 & 6 & Miller et al. (2008) \\
\hline NGC 2632 & 142 & 0.60 & 34 & 130 & 2 & Pepper et al. (2008); WEBDA \\
\hline NGC 2660 & 3000 & 1.00 & 19 & 150 & None & von Braun et al. (2004); WEBDA \\
\hline NGC 2682 & - & 4.50 & 18 & 35 & In progress & Quirrenbach et al. (2000); WEBDA \\
\hline NGC 6208 & 5000 & 1.00 & 22 & 61 & None & Lee et al. (2004) \\
\hline NGC 6633 & 2000 & 0.43 & 186 & 131 & None & Hidas et al. (2005) \\
\hline NGC 6791 & 3580 & 8.00 & 7 & 56 & "A few" & Bruntt et al. (2003) \\
\hline NGC 6791 & 650 & 8.00 & 84 & $>300$ & None & Mochejska et al. (2005) \\
\hline NGC 6791 & 3311 & 8.00 & 28 & 170 & None & Montalto et al. (2007); WEBDA \\
\hline NGC 6819 & - & 2.50 & 50 & 568 & In progress & Quirrenbach et al. (2000) \\
\hline NGC 6819 & 276 & 2.50 & 19 & 133 & 8 & Street et al. (2003) \\
\hline NGC 6940 & 50000 & 0.72 & 18 & 22 & 2 & Hood et al. (2005) \\
\hline NGC 7086 & 1000 & 0.60 & 12 & 60 & None & Rosvick \& Robb (2006) \\
\hline NGC 7789 & - & 1.70 & 53 & 218 & In progress & Quirrenbach et al. (2000) \\
\hline NGC 7789 & 2400 & 1.70 & 30 & 73 & 3 & Bramich et al. (2005) \\
\hline
\end{tabular}

A further simplification can be made by using the mass-radius relation for mainsequence stars. From Table 15.8 of Cox (2000) the mass-radius relation for main sequence stars (in the spectral type range F0 to M5) can be written approximately as $R_{*} \simeq M^{0.81}$ in solar units. Combining this with equation 2 gives

$$
P_{T}=R_{\odot}\left(\frac{M_{*}^{0.81}}{M_{*}^{1 / 3}}\right) p^{-2 / 3},
$$

where $R_{\odot}=0.00465 A . U$.. The quantity in parentheses in this equation is roughly equal to $M_{*}^{1 / 2}$, so in a population of $N_{*}$ stars, the expected number of transiting hot Jupiter planets can be found by multiplying $N_{*}$ by equations 1.1 and 1.3 :

$$
N_{\text {trH } J}=0.0003 N_{*} 10^{2.0[\mathrm{Fe} / \mathrm{H}]} M_{*}^{1 / 2} p^{-2 / 3} d p,
$$

where $\mathrm{p}$ has been converted from years to days. Equation 1.4 predicts about 6 transiting planets per 10000 solar-mass, solar-metallicity stars with periods near 3 days (taking dp $=4$ ). For comparison, in the Fischer and Valenti 2005 sample of 1040 stars with Doppler measures there is one transiting planet. Only two Doppler-discovered planets also transit. So there are no more than a few transiting planets per ten thousand stars.

\section{NGC 7789 and the future}

For about 12 years, we have been monitoring several old open clusters, searching for low-amplitude variability of cluster stars, using the Mt. Laguna Observatory 1-meter and the Lowell Observatory 1.83-meter Perkins Telescope. The original purpose of the project was to investigate photospheric stellar activity as a function of stellar age, but an obvious additional goal is to search for transiting planets. We report here on a preliminary evaluation of our data for possible transits in the 2-Gyr old cluster, NGC 7789.

Our observing cadence was not ideal for finding transits. For the stellar activity project we needed an hour or two of observing per cluster on a daily or weekly timescale, whereas for transits several hours of observing on a single cluster per night would be more appropriate. Nevertheless, we do have a large amount of data for the cluster. This 
Table 2. Potential Target Clusters for Future Transit Searches.

\begin{tabular}{|c|c|c|c|c|c|}
\hline Cluster & $\begin{array}{l}\text { Age } \\
{[\mathrm{Gyr}]}\end{array}$ & {$[\mathrm{Fe} / \mathrm{H}]$} & $\begin{array}{l}\text { \# of stars } \\
(\mathrm{V}<20)\end{array}$ & $\begin{array}{l}\text { Estimated \# of } \\
\text { transiting planets }\end{array}$ & References \\
\hline NGC 188 & 6.8 & 0.00 & 1400 & 0.73 & Sarajedini et al. (1999) \\
\hline NGC 1245 & 1.0 & -0.05 & 1300 & 0.53 & Burke et al. (2004) \\
\hline NGC 2099 & 0.4 & 0.08 & 1500 & 0.97 & Nilakshi \& Sagar (2002); WEBDA \\
\hline NGC 2141 & 2.5 & -0.26 & 2000 & 0.31 & Rosvick (1995) \\
\hline NGC 2158 & 2.0 & -0.60 & 2200 & 0.08 & Carraro et al. (2002) \\
\hline NGC 2168 & 0.2 & -0.3 & 800: & 0.11 & Barrado y Navascues et al. (2001) \\
\hline NGC 2194 & 0.6 & -0.27 & 1800 & 0.27 & Kyeong et al. (2005) \\
\hline NGC 2204 & 1.6 & -0.35 & 1100 & 0.11 & Kassis et al. (1997) \\
\hline NGC 2243 & 4.5 & -0.44 & 1900 & 0.13 & Kaluzny et al. (1996); WEBDA \\
\hline NGC 2477 & 1.0 & -0.05 & 1800 & 0.74 & Kassis et al. (1997) \\
\hline NGC 2682 & 4.0 & -0.05 & 600 & 0.25 & Montgomery et al. (1993) \\
\hline Cr 261 & $9-11$ & -0.14 & 1000 & 0.27 & Gozzoli et al. (1996) \\
\hline NGC 6253 & 3 & +0.3 & 600 & 1.24 & Bragaglia et al. (1997) \\
\hline NGC 6705 & 0.25 & 0.0 & 5000: & 2.59 & Sung et al. (1999) \\
\hline NGC 6791 & 8.0 & +0.3 & 5400 & 11.14 & Stetson et al. (2003) \\
\hline NGC 6819 & 2.4 & -0.05 & 1500 & 0.61 & Rosvick \& Vandenberg (1998) \\
\hline NGC 6939 & 1.3 & 0.00 & 500 & 0.25 & Andreuzzi, et al. (2004) \\
\hline NGC 7789 & 1.6 & -0.08 & 2500 & 0.90 & Gim et al. (1998); This paper \\
\hline
\end{tabular}

preliminary analysis of NGC 7789 is based on 159 nights of V-magnitude CCD images of the cluster.

We used a simple BLS procedure to search for transits. Because of our sparse sampling over a long period, the program found a large number of false positives, but none that convincingly could be called a candidate transit. We did find a number of other types of variables, and we are working to improve our search technique.

But should we expect to find any transiting planets? We used two approaches to address this question. Using equation 1.4 with $N_{*}=2000,[\mathrm{Fe} / \mathrm{H}]=-0.08, M_{*}=1, p=3$ and $d p=4$, we find that $N_{t r H J}$ is about 0.8 , that is, there are probably no planets.

We also tried a Monte-Carlo approach following a procedure by Weldrake \& Sackett (2005). We calculated a quantity, $P_{\text {window }}$, the probability for two or more transits at times of our observations, assuming that there is a hot Jupiter system in the cluster.

To calculate $P_{\text {window }}$, we tried a large number of model transit light curves with random periods, following the period distribution of hot Jupiters. We modeled transit durations in hours with $d_{\text {model }}=1.412 M^{-1 / 3} R_{*} p^{1 / 3} \simeq 1.412 p^{1 / 3}$, using one solar-mass, one solarradius stars. The mean value of $P_{\text {window }}$ for all the trials was 0.85 . So if there is a transiting planet in the cluster, we had an $85 \%$ probability of seeing two of its transits.

What about the future? What are the prospects for finding planets in open clusters? We have searched the WEBDA database for clusters that might be suitable targets for transit searches. We selected clusters with apparently large numbers of cluster members, based on the published photometry tabulated in the WEBDA. After downloading the photometry for a selected reference, we fitted a fiducial main sequence to the data and constructed a histogram of the numbers of stars between the main-sequence turnoff and magnitude 20 as a function of distance in (V-I) color index from the fiducial line. We then summed the numbers of stars in the histogram between 0.05 magnitudes to the blue side of the fiducial line and 0.2 magnitudes to the red side. This provided us with a relatively uniform evaluation of the number of main sequence stars in a cluster. However we have not taken into account the completeness of the original studies.

Table 2 displays the result of this data mining. We took the basic cluster information from the WEBDA or references listed in the table, and after finding the number of stars 
brighter than $\mathrm{V}=20$, we calculated the estimated number of transiting planets using equation 1.4. The total number of estimated cluster members is 32900 and the estimated number of transiting planets is 21.23. Individually, there are not enough potential transiting planets to be interesting, except possibly in NGC 6791. A massive effort would be required to search for planets in all, or even in the most promising clusters.

\section{References}

Andreuzzi, G., Bragaglia, A., Tosi, M., \& Marconi, G. 2004, MNRAS, 348, 297

Barrado y Navascues, D., Staufer, J. R., Bouvier, J., \& Martin, E. L 2001, ApJ, 546, 1006

Bragaglia, A., Tessicini, G., Tosi, M., Marconi, G., \& Munari, U. 1997, MNRAS, 284, 477

Bramich, D. M., Horne, K., Bond, I. A., Street, R. A., et al. 2005, MNRAS, 359, 1096

Bruntt, H., Grundahl, F., Tingley, B., Frandsen, S., et al. 2003, A\&SA, 410, 323

Burke, C. J., Gaudi, B. S., DePoy, D. L., \& Pogge, R. W. 2004, AJ, 127, 2382

Burke, C. J., Gaudi, B. S., DePoy, D. L., \& Pogge, R. W. 2006, AJ, 132, 210

Burke, C. J. 2008, http://www.astronomy.ohio-state.edu/ cjburke/STEPSS/

Carraro, G., Girardi, L., \& Marigo, P. 2002, MNRAS, 332, 705

Cox, A. N., Ed. 2000, Allen's Astrophysical Quantities 4th edition, pg. 389

Fischer, D. A. \& Valenti, J. 2005, ApJ, 622, 1102

Gilliland, R. L., Brown, T. M., Guhathakurta, P., Sarajedini, A., et al. 2000, ApJ, 545,47

Gim, M., Vandenberg, D. A., Stetson, P. B., Hesser, J. E., \& Zurek, D. R. 1998, PASP, 110, 1318

Gozzoli, E., Tosi, M., Marconi, G., \& Bragaglia, A. 1996, MNRAS, 283, 66

Hidas, M. G., Ashley, M. C. B., Webb, J. K., Irwin, M., et al. 2005, MNRAS, 360, 703

Hood, B., Cameron, A. C., Kane, S. R., Bramich, D. M., et al. 2005 MNRAS, 360,791

Howell, S. B., VanOutryve, C., Tonry, J. L., Everett, M. E., et al. 2005, PASP, 117, 1187

Janes, K. A., 1996, JGR, 101, 14853

Kaluzny, J., Krzeminski, W., \& Mazur, B. 1996, A\&AS, 118, 303

Kassis, M., Janes, K. A., Friel, E. D., \& Phelps, R. L. 1997, AJ, 113, 1723

Kyeong, J., Byun. Y.-I., \& Sung, E.-C. 2005, JKAS, 38, 415

Lee, B. L., von Braun, K., Mallen-Ornelas, G., Yee, H. K. C., Seager, S., \& Gladders, M. D. 2004, in SS. Holt \& D. Deming, (eds.), The Search for Other Worlds, AIP Conf. Proc. 713, (New York: AIP), p. 177

Miller, A. A., Irwin, J., Aigrain, S., Hodgkin, S., \& Hebb, L. 2008, MNRAS, 387, 349

Mochejska, B. J., Stanek, K. Z., Sasselov, D. D., Szentgyorgyi, A. H., et al. 2005, AJ, 129, 2856

Mochejska, B. J., Stanek, K. Z., Sasselov, D. D., Szentgyorgyi, A. H., et al. 2006, AJ, 131, 1090

Montalto, M., Piotto, G., Desidera, S., de Marchi, F., et al. 2007, A\&A, 470, 1137

Montgomery, K. A., Marschall, L. A., \& Janes, K. A. 1993, AJ, 106, 181

Nilakshi \& Sagar, R. 2002, A\&A A, 381, 65

Pepper, J., Stanek, K. Z., Pogge, R. W., Latham, D. W., et al. 2008, AJ, 135, 907

Quirrenbach, A., Cooke, J., Mitchell, D., Safizadeh, N. \& Deeg, H. in F. Garzon, C. Eiroa, D. de Winter, \& T. J. Mahoney (eds.), Disks, Planetesimals and Planets, ASP Conf. Proc. 219 (San Fransicso: ASP), p. 566.

Rosvick, J. M. 1995, MNRAS, 277, 1379

Rosvick, J. M. \& Robb, R. 2006, AJ, 132, 2309

Rosvick, J. M. \& Vandenberg, D. A. 1998, AJ, 115, 1516

Sarajedini, A., von Hippel, T., Kozhurina-Platais, V. \& Demarque, P. 1999, AJ, 118, 2894

Stetson, P. B., Bruntt, H., \& Grundahl, F. 2003, PASP, 115, 413

Street, R. A.,Home, K., Lister, T. A., Penny, A. J., et al. 2003, MNRAS, 340, 1287

Sung, H., Bessell, M. S., Park, B.-G., \& Kang, Y. H. 1999 MNRAS, 310, 982

von Braun, K., Lee, B. L., Malen-Ornelas, G., Yee, H. K. C., Seager, S., \& Gladders, M. D. in SS. Holt \& D. Deming, (eds.), The Search for Other Worlds, AIP Conf. Proc. 713, (New York: AIP), p. 181

Weldrake, D. T. F. \& Sackett, P. 2005, ApJ, 620, 1033 